\title{
Subregion holographic complexity and renormalization group flows
}

\author{
Pratim Roy ${ }^{*}$ and Tapobrata Sarkar ${ }^{\dagger}$ \\ Department of Physics, Indian Institute of Technology, Kanpur 208016, India
}

(Received 2 September 2017; published 30 April 2018)

\begin{abstract}
We investigate subregion holographic complexity in the context of renormalization group flow geometries. We use both the Poincaré slicing and the Janus ansatz as holographic duals to renormalization group flows in the boundary conformal field theory. In the former metric, subregion complexity is computed for a disc and a strip shaped entangling region. For the disc shaped region, consistent emergence of length scales for flow to the deep infrared is established. In the case of a strip shaped entangling region for both sharp and smooth domain walls, we compute the complexity and contrast its behavior with the holographic entanglement entropy. Finally, the complexity is computed numerically using the Janus ansatz.
\end{abstract}

DOI: 10.1103/PhysRevD.97.086018

\section{INTRODUCTION}

The statistics of information, popularly known as "information theory" is an old topic that has attracted great interest in diverse areas of science over the last few decades. Its more recent avatar, the quantum theory of information promises to be a leading character in the study of the quantum computer. While in its initial form, information theory was devised to deal with questions related to the "closeness" of statistical distributions; in quantum theories, it broadly attempts to understand the following question: How close are two quantum states? For pure states, this question was addressed in [1]. This was following an earlier work, which had dealt with a similar issue in the context of thermal states in an equilibrium thermodynamic system (for a review, see [2]). The thermal metric (characterizing the closeness of two equilibrium thermodynamic states) is known to be related [3] to the Fisher information metric, popularly studied in the statistics community. Broadly, the work of [1] developed a notion of a quadratic form, or a Riemannian metric on the space of parameters of the system where two pure quantum states are infinitesimally separated.

In the context of many body quantum systems at zero temperature, such discussions are relatively recent. Indeed, as is known by now, there are several measures of information in this context, one of them being the proposal of [4], which, given two quantum ground states in a many-body

\footnotetext{
*proy@iitk.ac.in

tapo@iitk.ac.in
}

Published by the American Physical Society under the terms of the Creative Commons Attribution 4.0 International license. Further distribution of this work must maintain attribution to the author(s) and the published article's title, journal citation, and DOI. Funded by SCOAP . system denoted by $\left|g_{1}\right\rangle$ and $\left|g_{2}\right\rangle$, the overlap function $\left|\left\langle g_{1} \mid g_{2}\right\rangle\right|$ provides a characterization of quantum phase transitions. In [5], the work of [4] was cast in a language similar to that of [1] and a "quantum information metric" was constructed for the transverse XY spin chain in a homogeneous magnetic field. It was shown that this metric (or more appropriately the scalar curvature associated to it) was indeed an effective measure to characterize quantum phase transitions in the model. A flurry of activity followed soon after, and the information metric and a related quantity known as the fidelity susceptibility were calculated in a variety of examples and remain two of the most popular information theoretic quantities (for a review, see [6]).

In quantum field theories, information theoretic studies have a long history, and it was realized sometime back that the renormalization group equations can be written in a geometric framework; see, e.g., [7-10]. A natural issue that then arises is the application of similar ideas in the context of string theory. While a study of overlaps between string states (in lines with [4]) might require a deeper understanding of string field theory and could be somewhat complicated, nonetheless, the anti-de Sitter/conformal field theory (AdS/CFT) correspondence provides a new approach to understanding the origins of quantum information in the context of quantum field theories, via gravity duals.

Indeed, there has been a recent upsurge of interest in using the AdS/CFT correspondence to elucidate several key concepts which are essentially quantum information theoretic in nature. Starting from the celebrated RyuTakayanagi (RT) conjecture for the entanglement entropy in quantum field theories [11], one such issue of current interest is the complexity of a quantum state. This quantity may roughly be defined as the least possible number of steps needed to construct the state from a given reference (thus capturing the "difficulty" in creating a quantum state). 
There are currently several proposals in the literature for calculating quantum complexity using the dual holographic route.

The first proposal, popularly known in the literature as the complexity $=$ volume $(\mathrm{CV})$ conjecture $[12,13]$, states that the complexity of a given quantum state on a time slice of the boundary CFT is dual to the volume of the maximal hypersurface in the bulk of codimension one, which is matched to the boundary at the given time. The second proposal is the complexity $=$ action (CA) conjecture [14]. This states that the holographic complexity can be evaluated by calculating the gravitational action on a Wheeler-de Witt patch of the bulk spacetime.

A related proposal, which will be the focus of this paper, was put forward in [15], based on the CV conjecture of [12]. It deals with holographic complexity (in the sense of a "reduced fidelity susceptibility") of subregions of the boundary CFT (for related works, see, e.g., [16-21]). This is, in some sense, a generalization of the CV conjecture to specific subregions in the boundary and relates to the volume of the bulk spacetime enclosed by a Ryu-Takayanagi minimal surface (called RT surface in the sequel) of codimension two. We will interchangeably call this the "complexity" or equivalently the "RT volume" in sequel.

The proposal of [15] for subregion holographic complexity reads

$$
\mathcal{C}_{\text {subregion }}=\frac{V(\gamma)}{8 \pi G L_{\mathrm{AdS}}}
$$

where $V(\gamma)$ is the volume of a minimal Ryu-Takayanagi surface, $G$ is the Newton's constant in appropriate dimension, and $L_{\mathrm{AdS}}$ is an AdS length scale. Various aspects of this quantity have been studied in the literature by now, including low and high temperature expansions in a thermal setting (see, e.g., [22]).

In this paper, we consider renormalization group (RG) flow scenarios in the context of subregion holographic complexity. For the CFT, adding a relevant operator triggers a RG flow to either an IR conformal fixed point, or the theory becomes massive. We study holographic RG flow geometries using both the Poincaré slicing and the Janus ansatz. Similar studies have been undertaken for the entanglement entropy in the past (see, for example, [23-25]). It is also relevant to mention that much of the work done in the context of the holographic entanglement entropy has been motivated by providing a holographic description for the $c$ functions and its proposed variants $[26,27]$. The Janus ansatz, which is the holographic dual of interface and boundary CFTS (ICFTS and BCFTs), has been treated in [25]. Although complexity does not naturally have an analogue of $c$ functions, this study is physically interesting in its own right. One can ask if, for example, what physical features of the system are captured by subregion complexity along RG flows. In particular, a natural question is whether complexity encodes information regarding holographic phase transitions (as discussed in [27]). It is also interesting to understand the emergence of different length scales in complexity along a RG flow, akin to what had been discussed in the context of entanglement entropy in [23]. Further, in line with a recent proposal of [28], this might provide useful information for geometric quantities along a RG flow.

With the above motivations in mind, in this paper we initiate a study of how holographic complexity behaves in a RG flow scenario. The paper is organized as follows: in Sec. II, we first compute the complexity for $d=3,4$ dimensional CFTs for a disc shaped entangling region (and assuming a sharp domain wall), for which the expressions are analytically simple and amenable to physical interpretation. We point out some differences between the complexity and the entanglement entropy in such a scenario and how different scales emerge in the context of the RG flows. We then proceed to calculate the complexity for an abrupt domain wall type of geometry for a strip shaped boundary subsystem and point out some of its aspects. It is known that this setup exhibits a phase transition for certain values of the parameters and we track the behavior of the complexity at this point. Next, we generalize to a smooth domain wall and numerically calculate the complexity and analyze the issue of phase transitions in the system. In Sec. III, we consider the Janus ansatz, and compute the complexity of ICFT and BCFT numerically. Finally, in Sec. IV, we conclude with a summary of results and future directions.

\section{HOLOGRAPHIC RG FLOW GEOMETRIES}

As mentioned in the Introduction, in the context of the AdS/CFT correspondence, a RG flow is triggered by a relevant operator in the CFT. The holographic dual to this operator is a bulk geometry which incorporates a scalar field. The resulting bulk action is given by (we follow the notations of [27])

$$
S=\frac{1}{2 \kappa^{2}} \int d^{(d+1)} x \sqrt{-g}\left(R-\frac{1}{2}(\partial \phi)^{2}-V(\phi)\right) .
$$

It is assumed that the scalar potential $V(\phi)$ has stationary points such that $\left.\frac{\delta V}{\delta \phi}\right|_{\phi_{i}}=0$ where the spacetime is AdS, with a negative potential energy $V\left(\phi_{i}\right)=-\frac{d(d-1)}{L^{2}} \alpha_{i}^{2}$. Here, $L$ is the fundamental length scale in the theory and $\alpha_{i}$ are dimensionless constants which are different for various fixed points. At the fixed points, the AdS curvature scale is given by $\tilde{L}=L / \alpha_{i}$. It is well known that the metric representing a holographic RG flow is given by [29]

$$
d s^{2}=d r^{2}+e^{2 A(r)} \eta_{i j} d x^{i} d x^{j} .
$$


In these coordinates, the UV is located at $r \rightarrow \infty$ and the IR is at $r \rightarrow-\infty$. At both the IR and the UV fixed points, the conformal factor $A(r)$ takes the simple form $\frac{r}{\tilde{L}}$. Note that the AdS length $\tilde{L}$ is different for the IR and the UV, with $L_{\mathrm{UV}}>L_{\mathrm{IR}}$. Of course, it is necessary to introduce a UV cutoff $r_{\infty}$ to obtain a finite result in holographic calculations. This cutoff has the expression $r_{\infty}=L_{\mathrm{UV}} \log \left(\frac{L_{\mathrm{UV}}}{\delta}\right)$, which is related to the standard cutoff $z_{\min }=\delta$ for the AdS metric in the Fefferman-Graham coordinates [27].

We note here that that the conditions $A(r) \sim r$ as $r \rightarrow \pm \infty$ and $A^{\prime \prime}(r) \leq 0$ (required to rule out further AdS boundaries [29]) are quite restrictive and one is limited to few consistent choices for $A(r)$. One of these is the step profile that we will consider in the next subsection, and an example of a continuous profile will be subsequently considered. We will now calculate the volume $V_{\Sigma}$ enclosed by the RT surface for various forms of the factor $A(r)$ and for various types of entangling surfaces. We begin with a disc shaped entangling region in a sharp domain wall geometry. Note that the sharp domain wall geometry is not an exact supergravity solution. Nonetheless, this is a good model to study as it offers an analytical handle which is difficult elsewhere.

\section{A. A sharp domain wall: Disc shaped entangling region}

The explicit form for the conformal factor $A(r)$ is taken as $[23,27]$

$$
A(r)=\left\{\begin{array}{ll}
A_{\mathrm{IR}}(r)=\frac{r-r_{0}}{L_{\mathrm{IR}}}+\frac{r_{0}}{L_{\mathrm{UV}}} & \text { for } r \leq r_{0} \\
A_{\mathrm{UV}}(r)=\frac{r}{L_{\mathrm{UV}}} & \text { for } r \geq r_{0}
\end{array} .\right.
$$

This is a step profile, which is continuous at $r=r_{0}$, the radius at which two AdS metrics with different curvatures are sewn together. We investigate the disc shaped entangling regions for the bulk metric of Eq. (3) starting with (a ball shaped region in) $\mathrm{AdS}_{5}$. We denote ${ }^{1}$ by $r=r(\rho)$ the embedding of the ball shaped region in $\mathrm{AdS}_{5}$, and take the entangling region to be $\rho \leq \ell$. Then the area of the RT surface that extends into the bulk in this case is given by

$$
\text { Area }=4 \pi \int_{0}^{\ell} d \rho \rho^{2} e^{3 A(r)}\left(1+e^{-2 A(r)} r^{\prime}(\rho)^{2}\right)^{\frac{1}{2}} .
$$

The solution to the equations of motion are obtained by minimizing this area functional. Introducing an $\mathrm{UV}$ cutoff $\delta$, which cuts off the integral for $r$ at $r_{\infty}=$ $-L_{\mathrm{UV}} \log \left(\delta / L_{\mathrm{UV}}\right)$, this solution is given by [23]

\footnotetext{
${ }^{1} \rho$ is a boundary radial coordinate here, and is not to be confused with the notation of the density matrix in the last section.
}

$r(\rho)= \begin{cases}r_{\mathrm{IR}}(\rho)=-\frac{L_{\mathrm{IR}}}{2} \log \left(\frac{\ell^{2}+\delta^{2}-\rho^{2}+c_{\mathrm{IR}}^{2}}{L_{\mathrm{IR}}^{2}}\right) & \text { for } \rho \leq \rho_{0} \\ r_{\mathrm{UV}}(\rho)=-\frac{L_{\mathrm{UV}}}{2} \log \left(\frac{\ell^{2}+\delta^{2}-\rho^{2}}{L_{\mathrm{UV}}^{2}}\right) & \text { for } \rho>\rho_{0},\end{cases}$

where $r\left(\rho_{0}\right)=r_{0}$ and position of the domain wall is given by

$$
\rho_{0}=\sqrt{\ell^{2}+\delta^{2}-L_{\mathrm{UV}}^{2} e^{-2 r_{0} / L_{\mathrm{UV}}}}
$$

The constant $c_{\mathrm{IR}}=\sqrt{L_{\mathrm{IR}}^{2} e^{-2 r_{0} / L_{\mathrm{IR}}}-L_{\mathrm{UV}}^{2} e^{-2 r_{0} / L_{\mathrm{UV}}}}$ is determined by the requirement $r_{\mathrm{UV}}\left(\rho_{0}\right)=r_{\mathrm{IR}}\left(\rho_{0}\right)$. We find that, as in the case of the entanglement entropy in this setup, the RT volume comes out in terms of some combinations of parameters, and for convenience, we define an effective length scale

$\ell_{\mathrm{eff}}=\sqrt{\ell^{2}-L_{\mathrm{UV}}^{2} e^{-2 r_{0} / L_{\mathrm{UV}}}} \equiv \sqrt{\ell^{2}-\ell_{\mathrm{cr}}^{2}+\mathcal{O}\left(\delta^{2}\right)}$

which is reminiscent of an effective radius of the entangling disc as seen from the IR, with $\ell_{\mathrm{cr}}^{2}=L_{\mathrm{UV}}^{2} e^{-2 r_{0} / L_{\mathrm{UV}}}+\mathcal{O}\left(\delta^{2}\right)$ being the minimum disc radius for the minimal surfaces to penetrate into the IR [23]. The equation which gives the expression for the volume is given schematically as

$$
V_{\Sigma}=4 \pi \int_{0}^{\ell} d \rho \rho^{2} \int_{r(\rho)}^{r_{\infty}} d r e^{3 A(r)}
$$

Recall at this point that the definition of the conformal factor [Eq. (4)] and the subsequent solutions for the profile of the RT surface are given by $r(\rho)$ [see Eq. (6)]. The volume $V_{\Sigma}$ is the sum of contributions from both the UV and IR regions, which are evaluated as separate terms,

$$
\begin{aligned}
V_{\Sigma}= & 4 \pi \int_{0}^{\rho_{0}} d \rho \rho^{2}\left[\int_{r_{\mathrm{IR}}(\rho)}^{r_{0}} d r e^{3 A_{\mathrm{IR}}(r)}+\int_{r_{0}}^{r_{\infty}} d r e^{3 A_{\mathrm{UV}}(r)}\right] \\
& +4 \pi \int_{\rho_{0}}^{\ell} d \rho \rho^{2} \int_{r_{\mathrm{UV}}(\rho)}^{r_{\infty}} d r e^{3 A_{\mathrm{UV}}(r)} .
\end{aligned}
$$

Now, the volume as computed from Eq. (10) evaluates to a lengthy expression, which is best presented by defining ${ }^{2}$ $\tilde{\epsilon}=L_{\mathrm{IR}} e^{-r_{0} / L_{\mathrm{IR}}}$, in which case it reads, in terms of $\ell_{\text {eff }}$ of Eq. (8),

\footnotetext{
${ }^{2}$ As explained in [23], $\tilde{\epsilon}$ plays a similar role as the UV cutoff $\delta$; however, it does not necessarily have to be small.
} 


$$
\begin{aligned}
V_{\Sigma}= & \frac{2 \pi^{2} L_{\mathrm{UV}}^{4}}{3}+\frac{4 \pi L_{\mathrm{UV}}^{4}}{9}\left(\frac{\ell}{\delta}\right)^{3}-\frac{4 \pi L_{\mathrm{UV}}^{4}}{3}\left(\frac{\ell}{\delta}\right) \\
& -\frac{4 \pi L_{\mathrm{UV}}^{4}}{9}\left(\frac{\ell_{\mathrm{eff}}}{\ell_{\mathrm{cr}}}\right)^{3}+\frac{4 \pi}{9} L_{\mathrm{IR}}^{4}\left(\frac{\ell_{\mathrm{eff}}}{\tilde{\epsilon}}\right)^{3}+\frac{4 \pi}{3} L_{\mathrm{UV}}^{4}\left(\frac{\ell_{\mathrm{eff}}}{\ell_{\mathrm{cr}}}\right) \\
& -\frac{4 \pi}{3} L_{\mathrm{IR}}^{4}\left(\frac{\ell_{\mathrm{eff}}}{\tilde{\epsilon}}\right) \\
& -\frac{4 \pi}{3} L_{\mathrm{UV}}^{4} \tan ^{-1}\left(\frac{\ell_{\mathrm{eff}}}{\ell_{\mathrm{cr}}}\right)+\frac{4 \pi}{3} L_{\mathrm{IR}}^{4} \tan ^{-1}\left(\frac{\ell_{\mathrm{eff}}}{\tilde{\epsilon}}\right)+\mathcal{O}(\delta) .
\end{aligned}
$$

We also record here the volume enclosed by the RT surface in the case when the geodesics do not penetrate into the IR, i.e., lie purely in the UV. In this case, it is easy to show that this volume is simply the first line of Eq. (11), i.e.,

$$
V_{\Sigma}^{\mathrm{UV}}=\frac{2 \pi^{2} L_{\mathrm{UV}}^{4}}{3}+\frac{4 \pi L_{\mathrm{UV}}^{4} \ell^{3}}{9 \delta^{3}}-\frac{4 \pi L_{\mathrm{UV}}^{4} \ell}{3 \delta} .
$$

Expectedly, this is also the result for $\ell_{\text {eff }}=0$, i.e., when the geodesics do not penetrate into the IR.

There are a couple of things to be noted here. First, the divergence structure of the volume in the above expression is $\sim \mathcal{O}\left(\delta^{-3}\right)+\mathcal{O}\left(\delta^{-1}\right)$, which is as expected for a bulk $\mathrm{AdS}_{5}$, and gives the familiar "volume law" (the strongest divergence structure). Second, let us consider the limit $\ell_{\text {eff }} / \tilde{\epsilon} \gg 1$; i.e., the effective length is much greater than the IR cutoff. Then, it is not difficult to see that the terms involving $L_{\mathrm{IR}}$ in Eq. (11) precisely go over to their UV counterparts in the first line of that equation, with the replacement $(\ell / \delta) \rightarrow\left(\ell_{\text {eff }} / \tilde{\epsilon}\right)$. This is what one would obtain if one is purely in the IR.

Before we end this subsection, we briefly comment on the case of bulk $\mathrm{AdS}_{4}$. A similar calculation for $\mathrm{AdS}_{4}$ yields (with $\ell_{\mathrm{cr}}, \ell_{\text {eff }}$, and $\tilde{\epsilon}$ being defined in the same way as in the $\mathrm{AdS}_{5}$ case)

$$
\begin{aligned}
V_{\Sigma}= & \frac{\pi L_{\mathrm{UV}}^{3}}{2}\left(\frac{\ell}{\delta}\right)^{2}-\pi L_{\mathrm{UV}}^{3} \log \left(\frac{\ell_{\mathrm{cr}}}{\delta}\right) \\
& -\frac{\pi L_{\mathrm{UV}}^{3}}{2}\left(\frac{\ell_{\mathrm{eff}}}{\ell_{\mathrm{cr}}}\right)^{2}+\frac{\pi L_{\mathrm{IR}}^{3}}{2}\left(\frac{\ell_{\mathrm{eff}}}{\tilde{\epsilon}}\right)^{2} \\
& -\frac{\pi}{2} L_{\mathrm{IR}}^{3} \log \left(1+\frac{\ell_{\mathrm{eff}}^{2}}{\tilde{\epsilon}^{2}}\right)
\end{aligned}
$$

and the RT volume for geodesics that lie purely in the UV region and does not see the domain wall is a purely divergent piece,

$$
V_{\Sigma}^{\mathrm{UV}}=\frac{\pi}{2} L_{\mathrm{UV}}^{3}\left(\frac{\ell}{\delta}\right)^{2}-\pi L_{\mathrm{UV}}^{3} \log \left(\frac{\ell}{\delta}\right) .
$$

Let us note some salient features of these equations as well. The volume law is again satisfied, as expected. Next, we note that for $\ell_{\text {eff }}=0$, the pure UV contribution matches with the first line of Eq. (13). Then, we consider the case $\ell / \ell_{\text {cr }} \sim 1$. Again, if we assume in the spirit of our previous discussion that $\ell_{\text {eff }} / \tilde{\epsilon} \gg 1$, then we see that the terms involving $L_{\mathrm{IR}}$ are again similar in form to their UV cousins in the first line of Eq. (13), with the replacement $(\ell / \delta) \rightarrow\left(\ell_{\text {eff }} / \tilde{\epsilon}\right)$.

\section{B. A sharp domain wall: Strip shaped entangling region}

We now briefly consider a sharp domain wall with a strip shaped entangling region. In this subsection, we will restrict ourselves to a bulk $\mathrm{AdS}_{3}$, and take the entangling surface to be a strip of length $\ell$. This geometry was considered in [27], where it was shown that this geometry shows an interesting phase behavior with a holographic phase transition (as a function of the strip length). In the initial part of this subsection, we briefly review the results contained therein. We remind the reader that the conformal factor $A(r)$ is taken to be of the form of Eq. (4) with the location of the domain wall at $r=r_{0}$, and as mentioned in the previous subsections, this profile is not a solution of the Einstein's equations resulting from Eq. (2), but we use it here as a toy model in conjunction with a strip shaped entangling region. We record the expression for the area functional,

$$
A=\int_{0}^{(\ell-\epsilon) / 2} d x \sqrt{\left(r^{\prime}\right)^{2}+e^{2 A(r)}} .
$$

The fact that the integrand does not depend on $x(r)$ translates into

$$
\frac{d x}{d r}=\frac{e^{-2 A(r)}}{\sqrt{K_{2}^{2}-e^{-2 A(r)}}}
$$

where we will consider only the branch for which the derivative of Eq. (16) is positive. Here, $K_{2} \equiv K_{2}(\ell)$ is a conserved quantity that is determined by demanding $x=0$ at $r=r_{*}$, which is the turning point of the minimal surface. There are two types of minimal surfaces for the geometry denoted by Eqs. (3) and (4), namely, those surfaces that stay purely in the UV region and those that penetrate deeper into the bulk and reach the IR region. For the ones that are entirely in the UV region, Eq. (16) is integrated to give

$$
x(r)=L_{\mathrm{UV}} \sqrt{K_{\mathrm{UV}}^{2}-e^{-2 r / L_{\mathrm{UV}}}} .
$$

According to the boundary condition mentioned before, $K_{\mathrm{UV}}=e^{-r_{*} / L_{\mathrm{UV}}}$. One also demands that $x=\ell / 2$ as $r \rightarrow \infty$, so that $\ell=2 L_{\mathrm{UV}} e^{-r_{0} / L_{\mathrm{UV}}}$. Since the turning point of the minimal surface lies in the UV region, we may define a relation $\ell \leq \ell_{2}$, where $\ell_{2}=2 L_{\mathrm{UV}} e^{-r_{0} / L_{\mathrm{Uv}}}$. 
The second, and more interesting class of surfaces, penetrate into the IR, implying that $r_{*}<r_{0}$. The conserved quantity in this case is

$$
K_{2}=K_{\mathrm{IR}}=e^{-A_{\mathrm{IR}}\left(r_{*}\right)} .
$$

The value of $x$ for which the minimal surface encounters the domain wall is denoted by, $x_{t}=x_{\mathrm{IR}}\left(r_{0}\right)=x_{\mathrm{UV}}\left(r_{0}\right)$. The parts of the geodesic lying in the UV and IR regions ( $x_{\mathrm{UV}}$ and $x_{\mathrm{IR}}$, respectively) may readily be found by directly integrating Eq. (16) to obtain

$$
\begin{aligned}
x_{\mathrm{IR}} & =L_{\mathrm{IR}} \sqrt{K_{\mathrm{IR}}^{2}-e^{-2 A_{\mathrm{IR}}(r)}}, \\
x_{\mathrm{UV}} & =L_{\mathrm{UV}} \sqrt{K_{\mathrm{IR}}^{2}-e^{-2 A_{\mathrm{UV}}(r)}}+c_{\mathrm{UV}} .
\end{aligned}
$$

Here, $c_{\mathrm{UV}}$ is an integration constant. By imposing the conditions, $x_{\mathrm{IR}}\left(r_{*}\right)=0$ and $x_{\mathrm{UV}}(r)=\ell / 2, c_{\mathrm{UV}}$ is found to be

$$
c_{\mathrm{UV}}=\frac{\ell}{2}-L_{\mathrm{UV}} K_{\mathrm{IR}}
$$

Expressions for the length of the strip and location $x_{t}$ of the domain wall may be found from the above equations and give

$$
\begin{aligned}
\ell & =2 L_{\mathrm{UV}} K_{\mathrm{IR}}-2\left(L_{\mathrm{UV}}-L_{\mathrm{IR}}\right) \sqrt{K_{\mathrm{IR}}^{2}-e^{-2 r_{0} / L_{\mathrm{UV}}}} \\
x_{t} & =\frac{L_{\mathrm{IR}}}{L_{\mathrm{UV}}-L_{\mathrm{IR}}}\left(L_{\mathrm{UV}} K_{\mathrm{IR}}-\frac{\ell}{2}\right) .
\end{aligned}
$$

From this, we may solve for $K_{\mathrm{IR}}$ to obtain

$$
K_{\mathrm{IR} \pm}=\frac{L_{\mathrm{UV}} \ell \pm \sqrt{\ell^{2}-4 L_{\mathrm{IR}}\left(2 L_{\mathrm{UV}}-L_{\mathrm{IR}}\right) e^{-2 r_{0} / L_{\mathrm{UV}}}}}{2 L_{\mathrm{IR}}\left(L_{\mathrm{UV}}-L_{\mathrm{IR}}\right)} .
$$

It may be noted that for the $K_{\mathrm{IR}_{+}}$to have real roots, we must have $\ell \geq \ell_{\text {cr }}$ with

$$
\ell_{\mathrm{cr}} \equiv 2 \sqrt{L_{\mathrm{IR}}\left(2 L_{\mathrm{UV}}-L_{\mathrm{IR}}\right) e^{-2 r_{0} / L_{\mathrm{Uv}}}} .
$$

Here, $\ell_{\mathrm{cr}}$ is interpreted as the critical strip length so that the geodesics penetrate into the IR. This is similar to the critical length encountered for the disc shaped entangling region in the previous subsections.

From the above discussions, it follows that as far as the entanglement entropy is concerned, in the region $\ell_{\mathrm{cr}}<\ell<\ell_{2}$, there are three valid solutions of the entanglement entropy. The pure UV part of the entropy is valid for $\ell<\ell_{2}$, whereas for $\ell>\ell_{2}$, the root $K_{\mathrm{IR}+}$ corresponds to consistent solutions, with $K_{\mathrm{IR}-}$ being ruled out as $d x / d r$ becomes negative here.
With these necessary ingredients from [27], we will now set up the expression for the volume enclosed by the minimal RT surface given from Eq. (3). The volume is given by an expression analogous to Eq. (10), and reads

$$
\begin{aligned}
V_{\Sigma}= & \int d x d r e^{A(r)} \\
= & \int_{0}^{x_{t}} d x\left[\int_{r_{\mathrm{IR}(x)}}^{r_{0}} d r e^{A_{\mathrm{IR}}(r)}+\int_{r_{0}}^{r_{\infty}} d r e^{A_{\mathrm{UV}}(r)}\right] \\
& +\int_{x_{t}}^{(\ell-\epsilon) / 2} d x \int_{r_{\mathrm{UV}(x)}}^{r_{\infty}} d r e^{A_{\mathrm{UV}}(r)} .
\end{aligned}
$$

Here, the UV cutoff $r_{\infty}=L_{\mathrm{UV}} \log \left(L_{\mathrm{UV}} / \delta\right)$. Note that we are using a separate cutoff $\epsilon$ for $x$, which may simply be related to the UV cutoff $\delta$ by the relation $r(x=(\ell-\epsilon) / 2)=r_{\infty}$ yielding [27]

$$
\epsilon=\frac{\delta^{2}}{L_{\mathrm{UV}} K_{\mathrm{IR}}} .
$$

It is easier to present the results by setting, without loss of generality, $r_{0}=0$, and with this, the expression for the complexity reads

$$
\begin{aligned}
V_{\Sigma}= & \frac{L_{\mathrm{UV}}^{2} \ell}{2 \delta}+\frac{L_{\mathrm{IR}}}{2}\left(\ell-2 K_{\mathrm{IR}} L_{\mathrm{UV}}\right) \\
& +\left(L_{\mathrm{UV}}^{2}-L_{\mathrm{IR}}^{2}\right) \tan ^{-1} \\
& \times\left(\frac{2 K_{\mathrm{IR}} L_{\mathrm{UV}}-\ell}{\sqrt{\left(2 K_{\mathrm{IR}} L_{\mathrm{IR}}-\ell\right)\left(2 K_{\mathrm{IR}}\left(L_{\mathrm{IR}}-2 L_{\mathrm{UV}}\right)+\ell\right.}}\right) .
\end{aligned}
$$

Note that the divergent piece comes purely from the UV contribution (this can also be understood by evaluating the volume in the case that the geodesics do not penetrate into the IR). One can now use Eq. (22) to compute the complexity. Note that in the case of entanglement entropy, the pure UV contribution $\sim \log (\ell / \delta)$, so that one could consistently subtract out the divergent piece by considering, for example, the difference in the entropy for two different strip lengths. In our case, it is more useful to look at the finite part of the volume under the RT surface, by subtracting the divergent part from Eq. (26).

The behavior of the complexity can be easily gleaned by substituting the values of $K_{\mathrm{IR} \pm}$ from Eq. (22) in the finite part of Eq. (26). These are called $V_{\Sigma+}$ and $V_{\Sigma-}$ in sequel. From Eq. (22), it is seen that these match at $\ell=\ell_{\text {cr }}$ and that at $\ell=\ell_{2}, V_{\Sigma-}=0$. Also, it can be checked that $V_{\Sigma+}-V_{\Sigma-}$ is a monotonically increasing function in the interval $\ell_{\mathrm{cr}}<\ell<\ell_{2}$. From considerations of the entanglement entropy, it has been shown in [27] that there is a first order phase transition in the interval $\ell_{\text {cr }}<\ell<\ell_{2}$, when the entropy from the pure UV part equals that coming from the branch $K_{\mathrm{IR}+}$. Our discussion above, coupled with 
(a)

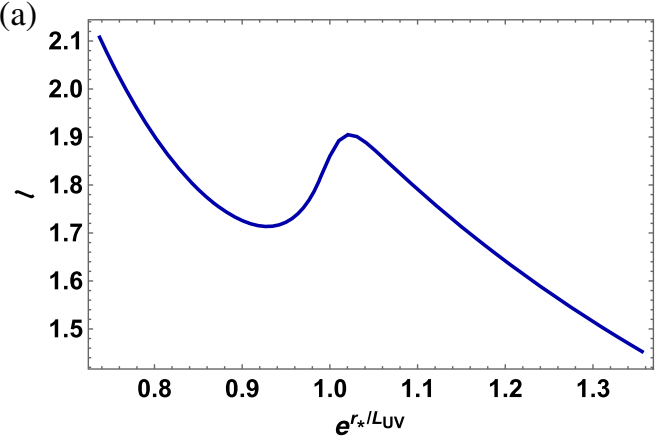

(b)

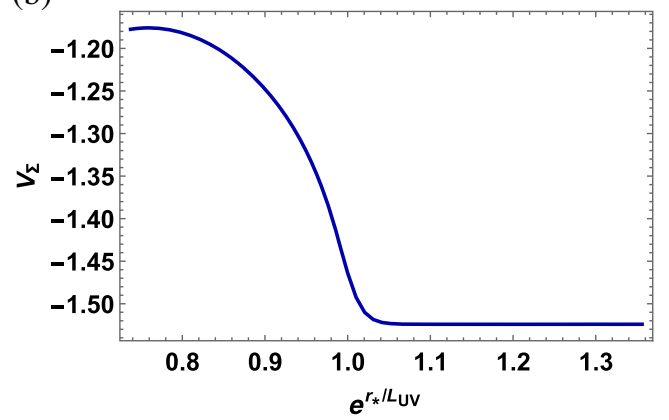

FIG. 1. Panel (a) $\ell$ is plotted as a function of $e^{r_{*} / L_{\mathrm{Uv}}}$. Panel (b) $V_{\Sigma}$ is plotted as a function of $e^{r_{*} / L_{\mathrm{Uv}}}$. Both (a) and (b) have the following parameter values: $L=0.66, \gamma=0.5 R, R=0.02$.

the fact that in this case, there is no nontrivial dependence of the complexity on the strip length in the pure UV region, leads to the conclusion that there is a discontinuous jump in the complexity at such a transition, but the precise location of this jump is determined from the holographic entanglement entropy.

\section{A smooth domain wall: Strip shaped entangling region}

So far, we have considered a sharp domain wall for simplicity. We now consider a more realistic profile for the warp factor $A(r)$ considered in [27], which has continuous interpolation between the IR and UV regions. Our aim in this subsection is to understand the nature of the RT volume for these theories and contrast it with the entanglement entropy. Similar to our previous example of a sharp domain wall, this solution also exhibits a phase transition, but in a more physically reasonable setup.

For a smooth domain wall, the functional form chosen for the profile is ${ }^{3}$

$$
e^{A(r)}=e^{r / L}(2 \cosh (r / R))^{-\gamma} .
$$

The AdS length scales at the UV and IR fixed points are given by

$$
\frac{1}{L_{\mathrm{UV}}}=\left(\frac{1}{L}-\frac{\gamma}{R}\right), \quad \frac{1}{L_{\mathrm{IR}}}=\left(\frac{1}{L}+\frac{\gamma}{R}\right)
$$

As before, the equation determining the minimal surfaces is given by

$$
\frac{d x}{d r}=\frac{e^{-2 A(r)}}{\sqrt{K_{2}^{2}-e^{-2 A(r)}}} .
$$

With the $A(r)$ chosen according to Eq. (27), an analytic solution to the above equation is no longer possible.

\footnotetext{
${ }^{3}$ The symbol $L$ used in Eq. (27) should not be confused with $L$ or $L_{\mathrm{UV} / \mathrm{IR}}$ used previously.
}

However, one determines that $x(r) \sim \sqrt{r-r_{*}}$ as $r \rightarrow r_{*}$. With this asymptotic behavior, we may develop a series solution of $x(r)$ in the neighborhood of the point $r=r_{*}$. The series solution can be treated as the initial condition for numerically finding the solution of $x(r)$ by shooting from a point close to $r=r_{*}$. To track the behavior of the complexity across a phase transition, it is necessary to plot the strip length $\ell$ as a function of $r_{*}$. We may obtain $\ell$ from the relation,

$$
\ell\left(r_{*}\right)=2 \lim _{r \rightarrow \infty} x(r) .
$$

As discussed in [27], specific choices of the parameters $L$, $\gamma$, and $R$ can produce different types of solutions for the strip length $\ell$. In particular, we will focus here on a choice of parameters that can produce multiple valued $\ell$ (for some range of $r_{*}$ ) as a function of $r_{*}$. It is known that such solutions of $\ell$ might indicate first order phase transitions in the theory, as a function of the strip length. In Fig. 1(a), we present such a solution, for $L=0.66, \gamma=0.5 R, R=0.02$ $\left(\gamma / R\right.$ is fixed). ${ }^{4}$ As we will see, the results are independent of the UV cutoff $\delta$. It may be seen from Fig. 1(a) that the specific values of the parameters chosen produce a kink in $\ell$ for a range of $r_{*}$. The physical consequence of this kink is that there are multiple values of $r_{*}$ that are valid for a particular value of $\ell$ within a certain range of the strip length. This, of course, is analogous to the range of $\ell$ in the previous subsection $\left(\ell_{\mathrm{cr}} \leq \ell \leq \ell_{2}\right)$ for which there are multiple choices of the minimal surface. In the present case, we need to determine the values of $\ell_{\mathrm{cr}}$ and $\ell_{2}$ numerically.

The values of $\ell_{\text {cr }}$ and $\ell_{2}$ can be determined from the solution of $\ell\left(r_{*}\right)$ by solving $\frac{d \ell}{d r_{*}}=0$ numerically. Having solved for $\ell_{\mathrm{cr}}$ and $\ell_{2}$, we are in a position to compute the volume numerically from the integral,

$$
V_{\Sigma}=\int_{r(x)}^{r_{\infty}} d r e^{A(r)} x(r)
$$

\footnotetext{
${ }^{4}$ For ease of comparison with existing literature, we choose the same values of the parameters as in [27].
} 

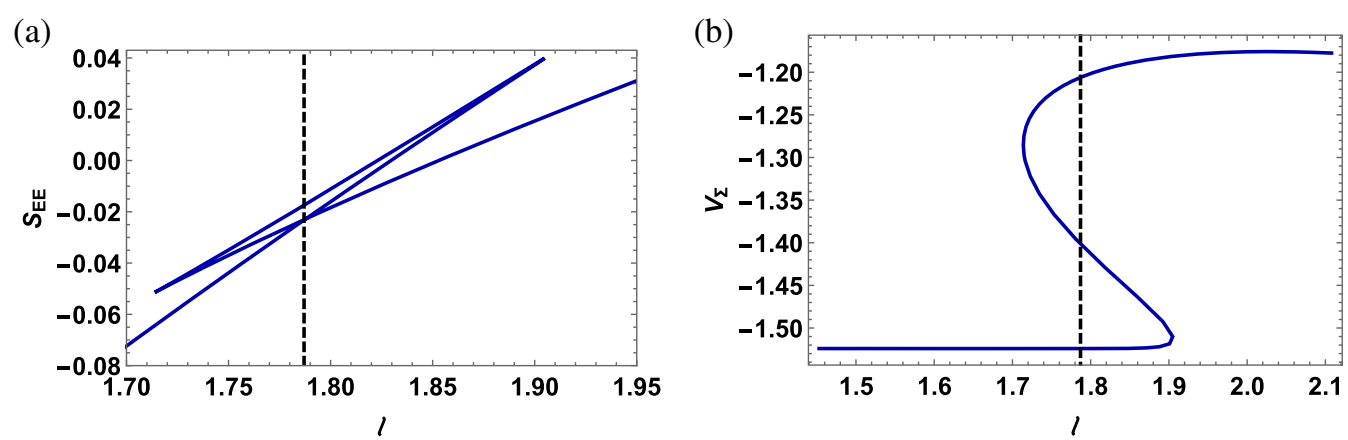

FIG. 2. Panel (a) Entanglement entropy is plotted as a function of $\ell$. Panel (b) $V_{\Sigma}$ is plotted as a function of $\ell$. Both (a) and (b) have the following parameter values: $L=0.66, \gamma=0.5 R, R=0.02$. The dashed vertical line denotes the point of phase transition $\ell \approx 1.79$.

The expression for the volume implicitly depends on $r_{*}$, since $\ell \equiv \ell\left(r_{*}\right)$. Now, since we have both $V_{\Sigma}$ and $\ell$ as a function of $e^{r_{*} / L_{\mathrm{UV}}}$ (which is a convenient scale for the $x$ axis), it is not difficult to express $V_{\Sigma}$ as a function of $\ell$. A word on regulating the volume is in order. It can be verified that the factor $A(r)$ behaves as $A(r) \sim \frac{r}{L}-\frac{\gamma r}{R}$ as we approach $r \rightarrow \infty$. Evaluating the above integral in the limit $r \rightarrow \infty$ (which is responsible for the UV divergence in the volume) yields the divergent contribution

$$
V_{\Sigma, \mathrm{div}}=\frac{\ell}{2} \frac{1}{\left(\frac{1}{L}-\frac{\gamma}{R}\right)} e^{r_{\infty}\left(\frac{1}{L}-\frac{\gamma}{R}\right)}
$$

that has to be subtracted out from Eq. (31) to obtain a finite expression. Indeed, this is the only divergence in the theory and has the expected $\ell / \delta$ behavior. There is therefore no problem in regulating the complexity (numerically) in a consistent manner throughout the flow. As we have mentioned, it may be observed from Fig. 1(a) that there are three possible branches of $r_{*}$ for a fixed value of $\ell$ (for a range of $\ell$ ). Figure 1(b) shows $V_{\Sigma}$ as a function of $e^{r_{*} / L_{\mathrm{Uv}}}$. Figures 2(a) and 2(b) show the entanglement entropy and the complexity as a function of $\ell$. In the case of the former, we see the typical swallow-tail behavior as observed for the sharp domain wall in [27]. As far as $V_{\Sigma}$ is concerned, it shows a multivalued behavior with respect to $\ell$. In this figure, the vertical dashed line denotes the phase transition, $\ell \approx 1.79$. As before, we see that the complexity shows a discontinuous jump across the phase transition.

\section{COMPLEXITY WITH THE JANUS ANSATZ}

Having computed the complexity for the RG flow in the Poincaré slicing, we now proceed to the Janus solution. The Janus solution was first introduced in the literature in [30] and was a nonsupersymmetric deformation of AdS resulting in a dual field theory which had a codimension one defect. The defect separated the field theory into two regions with different Yang-Mills couplings. Conformal field theories of this type which have an interface or a boundary have been extensively studied in the context of holography (see, for example, [25,31,32]). In the subsequent discussion, we shall mainly follow the conventions of [25].

The Janus solution is an ansatz where $\operatorname{AdS}_{d+1}$ is foliated using $\operatorname{AdS}_{d}$ slices. Let us take the Poincaré sliced metric of $\operatorname{AdS}_{d+1}$,

$$
d s^{2}=\frac{1}{z^{2}}\left(d z^{2}+d x_{\perp}^{2}-d t^{2}+\sum_{i=2}^{d-1} d x_{i}^{2}\right) .
$$

The above metric can be mapped to the Janus ansatz by the following transformation:

$$
x_{\perp}=y \cos \mu \quad z=y \sin \mu
$$

resulting in

$$
d s^{2}=\frac{1}{\sin ^{2} \mu}\left(d \mu^{2}+\frac{d y^{2}-d t^{2}+\sum_{i=2}^{d-1} d x_{i}^{2}}{y^{2}}\right) .
$$

In contrast to the Poincare sliced metric where the boundary is located at $z=0$, the above metric has three distinct components which can be interpreted as boundaries, namely, $\mu=0, \pi$ and $y=0$. We can interpret the $\mu=0, \pi$ as half-spaces corresponding to two different CFTs and the $y=0$ as the codimension one defect where the two half-spaces and joined together. Motivated by the prospect of studying how the complexity behaves in a boundary/interface CFT, we compute the volume of the Janus solution in the subsequent discussion, restricting ourselves to $d=2$.

Since we are interested in a RG flow scenario, we make the following slight change to the Janus ansatz:

$$
d s^{2}=f(\mu)\left(d \mu^{2}+\frac{d y^{2}-d t^{2}+\sum_{i=2}^{d-1} d x_{i}^{2}}{y^{2}}\right)
$$

where the factor $f(\mu)$ equals $f(\mu)=\frac{1}{\sin ^{2} \mu}$ at the fixed points where the space is AdS. To solve for the geometry numerically, we shall need the Einstein equations resulting 
from Eq. (2), with $\phi \equiv \phi(\mu)$. Plugging in the metric (36), one obtains the following equations [25]:

$$
\begin{array}{r}
\phi^{\prime \prime}-f \hat{V}^{\prime}(\phi)+\frac{d-1}{2} \frac{f^{\prime}}{f} \phi^{\prime}=0 \\
\frac{f^{\prime \prime}}{f}-\frac{3}{2} \frac{f^{\prime 2}}{f^{2}}+\frac{4}{d-1} \phi^{\prime 2}-2=0 \\
\frac{1}{4} \phi^{\prime 2}-\frac{d(d-1)}{32} \frac{f^{\prime 2}}{f^{2}}-\frac{d(d-1)}{8}+\frac{d(d-1)}{8} f-\frac{1}{2} f \hat{V}=0
\end{array}
$$

where we have written the potential as

$$
V(\phi)=-\frac{d(d-1)}{4}+\hat{V}(\phi)
$$

Out of the three equations in Eq. (37), we treat the first two as independent equations and the third as a constraint. While seeking solutions to the above equations that specify the geometry, we take a toy model for the potential of the form,

$$
\hat{V}(\phi)=\frac{1}{2} m^{2} \phi^{2}+\frac{1}{4 !} \lambda_{4} \phi^{4} .
$$

According to the holographic dictionary, the mass of the scalar field $m$ is related to the conformal dimension in the dual field theory as

$$
m^{2}=\Delta(\Delta-d)
$$

Since our motivation is to study RG flows, which flow to IR fixed points, we choose the conformal dimensions such that the scalar operator is relevant in the IR, i.e.,

$$
-\frac{d^{2}}{4}<m^{2}<0 \quad \frac{d}{2}<\Delta<d .
$$

In the $\mathrm{AdS}_{d}$ slicing, $\phi$ can be shown to have the near boundary behavior,

$$
\phi(\mu)=\alpha \mu^{\Delta}+\beta \mu^{d-\Delta}+\cdots
$$

This behavior is actually analogous to the well-known behavior of the scalar field, the Poincaré slicing [25], and $\alpha$ and $\beta$ may be identified with the expectation value and the source of the operator respectively.

With Eq. (37) fully specified, the initial conditions for $\phi(\mu)$ and $f(\mu)$ can now be specified. We take the boundary of one half-space to be located at $\mu=0$. As this is a fixed point in the RG flow, the space is in AdS and so $f(\mu)=\frac{1}{\mu^{2}}$ to leading order in $\mu$. Going beyond the leading order, the asymptotic behavior of the fields is given by [25]

$$
\begin{aligned}
& f(\mu)=\frac{1}{\mu^{2}}+\frac{1}{3}+\frac{1}{15} \mu^{2}-2 \beta^{2} \frac{\Delta-2}{2 \Delta-5} \mu^{2-2 \Delta}+\cdots \\
& \phi(\mu)=\beta \mu^{2-\Delta}-\frac{1}{12} \beta(\Delta-1) \mu^{4-\Delta}+\cdots
\end{aligned}
$$

It may be noted here that we have set the expectation value of the scalar field $\alpha=0$.

Similar to the calculation for entanglement entropy, we consider a strip entangling surface of length $\ell$. The minimal surfaces are those that intersect the constant time slice of the geometry

$$
d s_{\Sigma}^{2}=f(y)\left(d \mu^{2}+\frac{d y^{2}}{y^{2}}\right)
$$

and are parametrized by $y \equiv Y(\mu)$. The area functional is given by

$$
\text { Area }=\int d \mu \sqrt{f(\mu)\left(1+\frac{Y^{\prime}(\mu)^{2}}{Y(\mu)^{2}}\right)} .
$$

The geodesic equation that minimizes the above functional is given by

$$
f^{\prime} Y^{\prime}\left(Y^{2}+Y^{\prime 2}\right)+2 f Y\left(Y Y^{\prime \prime}-Y^{\prime 2}\right)=0
$$

Using the asymptotic behaviors of $f(\mu)$ and $\phi(\mu)$ from Eq. (43), we get the initial data for $Y(\mu)$ as

$$
Y(\mu)=\ell+\hat{y} \mu^{2}+\frac{\beta^{2} \hat{y}(\Delta-2)}{(\Delta-3)(2 \Delta-5)} \mu^{6-2 \Delta} .
$$

Now, we proceed to solve the set of Eqs. (37) and (47) numerically using a shooting method. The characteristics of the solutions are discussed at length in [25], but we include some brief remarks here for completeness. Whether the geometry is dual to an ICFT or a BCFT depends on the source strength $\beta$ of the scalar operator in the conformal field theory. It can be found that the solution blows up if the magnitude of $\beta$ is increased beyond a certain critical value (which, of course, depends on the two other constants which have to be fixed, namely, $\lambda_{4}$ and $\Delta$ ). Numerically, we monitor the value of $f(\mu)$ while integrating the system of equations. For a value of $\beta$ below the critical value, we find that $f(\mu)$ may again be approximated by $\frac{1}{\sin ^{2} \mu}$ at $\mu=\mu_{*}$, indicating that the geometry has flowed into another AdS region. This is thus an example of an ICFT. For values of $\beta$ greater than the critical value, $f(\mu)$ blows up at $\mu=\mu_{*}$, indicating that the theory has acquired a mass.

The geodesic solutions are also obtained from Eqs. (37) and (47) and it may be readily verified that for the BCFT geometry, which is singular at $\mu=\mu_{*}$, there is only one geodesic $Y(\mu)=\ell$, which reaches the singularity. All other geodesics are repelled and do not reach the singularity. 

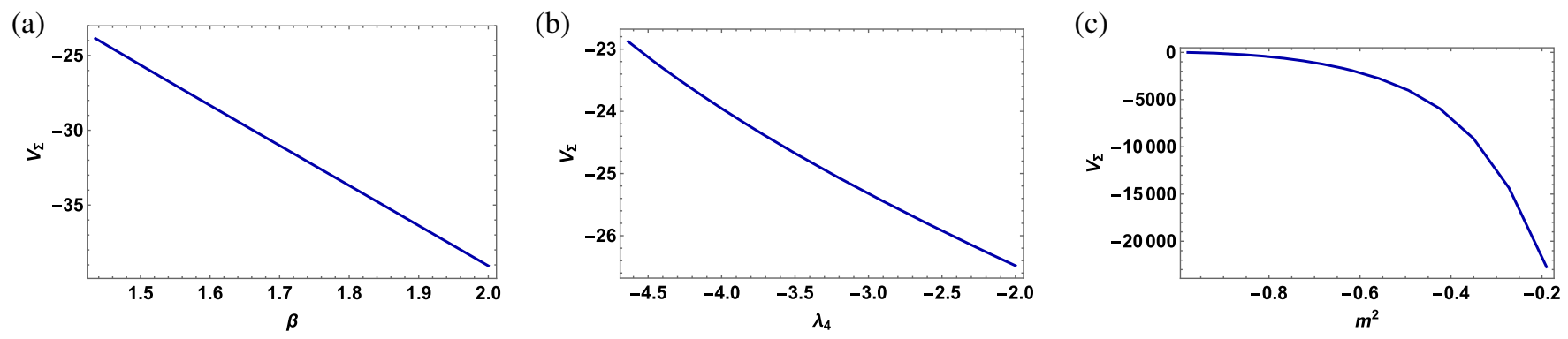

FIG. 3. $\quad V_{\Sigma}$ as a function of (a) $\beta$, (b) $\lambda_{4}$, and (c) $m^{2}$ for BCFTs. For (b) and (c), $\beta=1.4$.
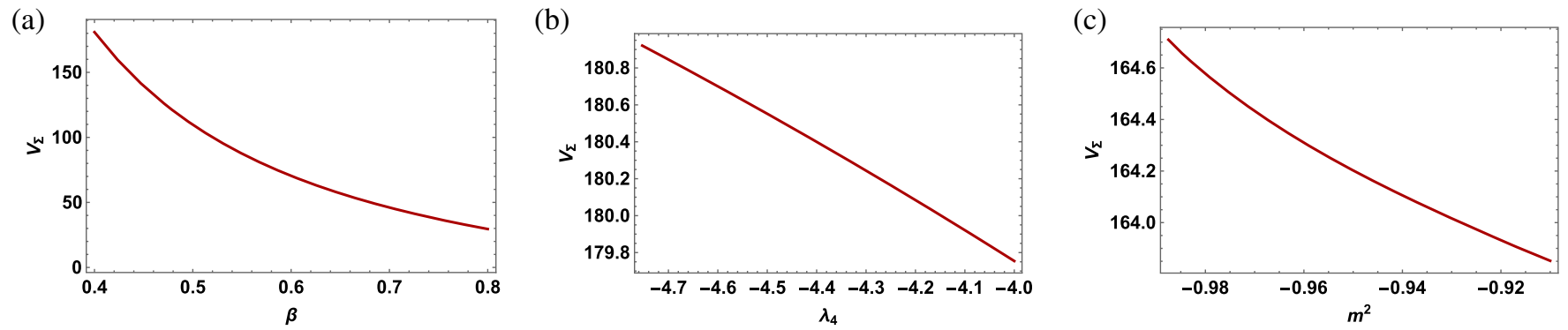

FIG. 4. $\quad V_{\Sigma}$ as a function of (a) $\beta$, (b) $\lambda_{4}$, and (c) $m^{2}$ for ICFTs. For (b) and (c), $\beta=0.4$.

So, this unique geodesic is the one which is used to calculate the complexity. In the ICFT case, of course, there is an infinite class of geodesics which reaches the point $\mu=\mu_{*}$.

With the numerical solutions of $f(\mu)$ and $Y(\mu)$ in hand, we proceed to calculate the complexity, which is given by

$$
V_{\Sigma}=\int_{\mu_{\epsilon}}^{\mu_{*}} d \mu f(\mu) \int_{\epsilon}^{Y(\mu)} \frac{d y}{y}
$$

Here, $\mu_{\epsilon}=\frac{\epsilon}{\ell}$ is the counterpart of the UV cutoff in the Poincaré slicing. Following $[33,34]$, we note that, strictly speaking, we should impose a separate cutoff (say $\delta$ ) for the coordinate $y$. However, since we concentrate on a purely numerical treatment (and moreover we have set $\ell=1$ throughout) it is not difficult to see that this does not affect any of our results below.

Similar to the entanglement entropy, the complexity is a divergent quantity due to the the behavior $f(\mu) \sim \frac{1}{\mu^{2}}$ as $\mu \rightarrow 0$. The expression for the volume may be regulated by subtracting out the divergent part, which may be evaluated to be $\frac{\ell}{\epsilon} \log \left(\frac{\ell}{\epsilon}\right)$. By inspection of Eq. (49), it is seen that the finite part of the complexity is independent of $\ell$.

Figures 3 and 4 show our numerical results. Here, we have plotted $V_{\Sigma}$ as a function of $\beta, \lambda_{4}$, and $m^{2}$. Note that the complexity is a monotonically decreasing function of $\beta$. This is similar in nature to the behavior of the entanglement entropy (or more appropriately the "g function") studied for BCFTs in $d=2$ [25].

\section{CONCLUSIONS AND DISCUSSIONS}

In this paper, we have initiated a study of subregion holographic complexity for renormalization group flow scenarios. We have considered two distinct cases here. First, we studied this quantity for a domain wall setup, both with a sharp and a smooth domain wall. Next, we computed the complexity for the case of the Janus solution.

Our computation of the complexity or the RT volume for a sharp domain wall scenario was carried out for both a disc shaped and a strip shaped entangling region. Indeed, this analysis revealed some interesting properties, for example the emergence of consistent length scales, which could be contrasted with the behavior of the entanglement entropy. For both the sharp as well as the smooth domain wall, we noticed that at the holographic phase transition (revealed by the analysis of [27]), the complexity undergoes a discontinuous jump. Finally, we computed the complexity for the Janus solution and obtained its variation with respect to the system parameters.

A deeper understanding of subregion complexity and its relation to the Fisher information metric is of great interest. We have taken the initial steps in this paper, and hope to report on further progress in a forthcoming work.

\section{ACKNOWLEDGMENTS}

We gratefully acknowledge the anonymous referee for pointing out an error in an initial draft of this paper, which led to modifications of some of the results. The work of T. S. is supported in part by Science and Engineering Research Board (India) via Project No. EMR/2016/008037. 
[1] J. Provost and G. Vallee, Riemannian structure on manifolds of quantum states, Commun. Math. Phys. 76, 289 (1980).

[2] G. Ruppeiner, Riemannian geometry in thermodynamic fluctuation theory, Rev. Mod. Phys. 67, 605 (1995).

[3] D. C. Brody and D. W. Hook, Information geometry in vapour-liquid equilibrium, J. Phys. A 42, 023001 (2009).

[4] P. Zanardi and N. Paunković, Ground state overlap and quantum phase transitions, Phys. Rev. E 74, 031123 (2006).

[5] P. Zanardi, P. Giorda, and M. Cozzini, InformationTheoretic Differential Geometry of Quantum Phase Transitions, Phys. Rev. Lett. 99, 100603 (2007).

[6] S.-J. Gu, Fidelity approach to quantum phase transitions, Int. J. Mod. Phys. B 24, 4371 (2010).

[7] M. Lassig, Geometry of the renormalization group with an application in two dimensions, Nucl. Phys. B334, 652 (1990).

[8] B. P. Dolan, A geometrical interpretation of renormalization group flow, Int. J. Mod. Phys. A 09, 1261 (1994).

[9] B. P. Dolan, Geodesic renormalization group flow, Int. J. Mod. Phys. A 12, 2413 (1997).

[10] B. P. Dolan, Renormalization group flow and geodesics in the $\mathrm{O}(\mathrm{N})$ model for large N, Nucl. Phys. B528, 553 (1998).

[11] S. Ryu and T. Takayanagi, Aspects of Holographic Entanglement Entropy, J. High Energy Phys. 08 (2006) 045.

[12] L. Susskind, Computational complexity and black hole horizons, Fortschr. Phys. 64, 24 (2016).

[13] L. Susskind, Addendum to computational complexity and black hole horizons, Fortschr. Phys. 64, 44 (2016).

[14] A. R. Brown, D. A. Roberts, L. Susskind, B. Swingle, and Y. Zhao, Holographic Complexity Equals Bulk Action?, Phys. Rev. Lett. 116, 191301 (2016).

[15] M. Alishahiha, Holographic complexity, Phys. Rev. D 92, 126009 (2015).

[16] O. Ben-Ami and D. Carmi, On volumes of subregions in holography and complexity, J. High Energy Phys. 11 (2016) 129.

[17] N. S. Mazhari, D. Momeni, S. Bahamonde, M. Faizal, and R. Myrzakulov, Holographic complexity and fidelity susceptibility as holographic information dual to different volumes in AdS, Phys. Lett. B 766, 94 (2017).

[18] M. K. Zangeneh, Y. C. Ong, and B. Wang, Entanglement entropy and complexity for one-dimensional holographic superconductors, Phys. Lett. B 771, 235 (2017).
[19] W.-C. Gan and F.-W. Shu, Holographic complexity: A tool to probe the property of reduced fidelity susceptibility, Phys. Rev. D 96, 026008 (2017).

[20] M. Flory, A complexity/fidelity susceptibility $g$-theorem for $\mathrm{AdS}_{3} / \mathrm{BCFT}_{2}$, J. High Energy Phys. 06 (2017) 131.

[21] D. Momeni, A. Myrzakul, R. Myrzakulov, M. Faizal, S. Alsaleh, and L. Alasfar, Thermodynamics of AdS black holes as regularized fidelity susceptibility, arXiv:1704 .05785 .

[22] P. Roy and T. Sarkar, Note on subregion holographic complexity, Phys. Rev. D 96, 026022 (2017).

[23] T. Albash and C. V. Johnson, Holographic entanglement entropy and renormalization group flow, J. High Energy Phys. 02 (2012) 095.

[24] H. Liu and M. Mezei, Probing renormalization group flows using entanglement entropy, J. High Energy Phys. 01 (2014) 098.

[25] M. Gutperle and J. Samani, Holographic RG-flows and Boundary CFTs, Phys. Rev. D 86, 106007 (2012).

[26] J. de Boer, M. Kulaxizi, and A. Parnachev, Holographic entanglement entropy in Lovelock gravities, J. High Energy Phys. 07 (2011) 109.

[27] R. C. Myers and A. Singh, Comments on holographic entanglement entropy and RG flows, J. High Energy Phys. 04 (2012) 122.

[28] S. Banerjee, J. Erdmenger, and D. Sarkar, Connecting Fisher information to bulk entanglement in holography, arXiv: 1701.02319

[29] D. Z. Freedman, S. S. Gubser, K. Pilch, and N. P. Warner, Renormalization group flows from holography supersymmetry and a c-theorem, Adv. Theor. Math. Phys. 3, 363 (1999).

[30] D. Bak, M. Gutperle, and S. Hirano, A dilatonic deformation of $\operatorname{AdS}(5)$ and its field theory dual, J. High Energy Phys. 05 (2003) 072.

[31] M. Gutperle and J.D. Miller, Entanglement entropy at holographic interfaces, Phys. Rev. D 93, 026006 (2016).

[32] K. Jensen and A. O'Bannon, Holography, Entanglement entropy, and conformal field theories with boundaries or defects, Phys. Rev. D 88, 106006 (2013).

[33] M. Gutperle and J.D. Miller, Entanglement entropy at holographic interfaces, Phys. Rev. D 93, 026006 (2016).

[34] D. Bak, A. Gustavsson, and S.-J. Rey, Conformal Janus on Euclidean sphere, J. High Energy Phys. 12 (2016) 025. 